\title{
Remarks on the spectral properties of tight binding and Kronig-Penney models with substitution sequences *
}

\author{
Anton Bovier ${ }^{\dagger}$ \\ Institut für Angewandte Analysis und Stochastik \\ Mohrenstrasse 39, D-10117 Berlin, Germany \\ and \\ Jean-Michel Ghez $\ddagger$ \\ Centre de Physique Théorique - CNRS Luminy, Case 907 \\ F-13288 Marseille Cedex 9, France \\ and PHYMAT, Département de Mathématiques \\ Université de Toulon et du Var, B.P. 132 \\ F-83957 La Garde Cedex, France
}

18 January 1994

\begin{abstract}
We comment on some recent investigations on the electronic properties of models associated to the Thue-Morse chain and point out that their conclusions are in contradiction with rigorously proven theorems and indicate some of the sources of these misinterpretations. We briefly review and explain the current status of mathematical results in this field and discuss some conjectures and open problems.
\end{abstract}

*Work partially supported by the Commission of the European Communities under contract No. SC1-CT91-0695

†e-mail: bovier@iaas-berlin.d400.de

‡e-mail: ghez@cpt.univ-mrs.fr 


\section{Introduction}

Following the discovery of quasicrystals by Shechtman et al. [1, 2] there has been a continuing interest of both physicists and mathematicians in structures that exhibit what has been termed deterministic disorder. A class of models that has attracted particular attention in this context are one dimensional Schrödinger operators with potentials obtained from so-called substitution sequences [3, 4] and a number of analytical and numerical tools have been developed for their investigation. On the other hand, substitution sequences provide examples of various types of aperiodic structures, e.g. quasiperiodic or not, that can be characterized by the nature of their Fourier spectrum which may be dense pure point (Fibonacci sequence), singular continuous (Thue-Morse sequence), or even absolutely continuous (RudinShapiro sequence). It is naturally of great interest to investigate the spectral and transport properties of systems in dependence of such properties. Let us mention that models based on substitution sequences have since enjoyed an increasing popularity also in different contexts such as one-dimensional quantum Ising chains [5, 6], aperiodically kicked quantum systems [7], etc.

About a decade has passed since the pioneering papers by Kohmoto et al.

[8] and Ostlund et al. 90 appeared and a vast amount of knowledge has since been accumulated through the work of both physicists and mathematicians and through methods ranging from numerical simulations, judicious guessing to heuristic and rigorous mathematics $110,11,12,13,14,115,16,17,18,19$, 20, 21, 22]. In spite of these efforts, we are today still far from a complete and coherent understanding of the properties of these systems which are, by and large, both subtle and unusual. This situation, together with the prospective technological applications (e.g. superlattices corresponding to substitution sequences can today be manufactured [23, 24]), invites a continued and coordinated effort to further research in this area.

A regrettable feature of the current situation is that in spite of a flourishing litterature and the existence of a number of review papers [2, 25, 26], the communication between different schools has been less than perfect and in particular it appears that what has been obtained in terms of mathematically rigorous results has not generally been recognized. As a result, there have appeared still very recently a number of papers in reknowned journals (see in particular [27, 28, 29, 30]) in which, on the basis of numerical and heuristic methods, results are claimed that are in striking contradiction to rigorously 
proven facts.

The present article is an attempt to improve this situation by explaining some of the mathematical results concerning the spectral theory of Schrödinger operators with substitution potentials and to rectify the errors in several recent papers that came to our attention. More importantly, we will try to explain the sources of the misinterpretations in these papers. Also, we would like to indicate, in an informal way that should be accessible to nonmathematicians, what mechanisms are important in leading to the mathematical results, what further results may be expected and what information would be needed to obtain these. We will also try to point to the serious open problems in the field. To a lesser extend, this article may also serve as a survey for the less involved reader.

Let us briefly review the types of models we consider. First we recall the definition of a substitution sequence. Take a finite set $\mathcal{A}$, called an alphabet and denote by $\mathcal{A}^{*}$ the set of all finitely long words that can be written in this alphabet. Moreover, we write $\mathcal{A}^{\mathbf{N}}$ and $\mathcal{A}^{\mathbf{Z}}$ for the sets of all semi-infinite and infinite sequences of letters from $\mathcal{A}$, respectively. Now let $\xi$ be a map from $\mathcal{A}$ to $\mathcal{A}^{*}$, i.e. a rule that associates to any letter in $\mathcal{A}$ a finite word. We call $\xi$ a substitution rule and extend it to a map from $\mathcal{A}^{*}$ to $\mathcal{A}^{*}$ by specifying that $\xi$ acts on a word by substituting each letter $\alpha_{i}$ of this word by its corresponding image $\xi\left(\alpha_{i}\right)$. By the same rule the action of $\xi$ is extended to $\mathcal{A}^{\mathbf{N}}$ and $\mathcal{A}^{\mathbf{Z}}$. A sequence $u \in \mathcal{A}^{\mathbf{N}}$ is then called a substitution sequence, if it is a fixpoint of $\xi$, i.e. if it remains invariant if each letter in the sequence is replaced by its image under $\xi$. Some simple conditions on $\xi$ assure the existence of such fixpoints. Moreover, except for trivial examples of substitution rules, this fixpoints are aperiodic sequences. Examples of substitution sequences that have attracted most attention in physics are

1. The Fibonacci sequence. Here $\mathcal{A}=\{a, b\}$ and the substitution rule is simply

$$
a \rightarrow \xi(a)=a b, \quad b \rightarrow \xi(b)=a
$$

2. The Thue-Morse sequence. Again $\mathcal{A}=\{a, b\}$, but the rule is this time

$$
a \rightarrow \xi(a)=a b, \quad b \rightarrow \xi(b)=b a
$$

3. The period-doubling sequence. Again $\mathcal{A}=\{a, b\}$, and the rule is

$$
a \rightarrow \xi(a)=a b, \quad b \rightarrow \xi(b)=a a
$$


4. The Rudin-Shapiro sequence. Here $\mathcal{A}=\{a, b, c, d\}$, and the rule is

$$
a \rightarrow \xi(a)=a c, \quad b \rightarrow \xi(b)=d c, \quad c \rightarrow \xi(c)=a b, \quad d \rightarrow \xi(d)=d b
$$

All of these examples, and in fact all substitutions we will consider have the property of being 'primitive' [4] which means, that there exists an integer $k$ such that for all pairs of letters $\alpha, \beta$ in $\mathcal{A}$, the word $\xi^{k}(\alpha)$ contains the letter $\beta$. Here $\xi^{k}$ means the $k$-fold application of $\xi$. In the sequel the term substitution will always be understood to mean 'primitive substitution'.

Given a substitution sequence, one may consider various associated Schrödinger operators. The most studied one is doubtlessly the tight binding model, where the Hamiltonian act on wave functions $\psi$ in the Hilbert space $\ell^{2}(\mathbf{Z})$ of square integrable sequences as

$$
H_{t b} \psi(n) \equiv \psi(n+1)+\psi(n-1)+v_{n} \psi(n)
$$

Here the potential $v_{n}$ is obtained by assigning real value $v(\alpha)$ to each letter in $\mathcal{A}$ and setting $v_{n}=v(\alpha)$, if the $n$-th letter in the substitution sequence $u$ is $\alpha$. Moreover, for negative values of $n$ one sets $v_{n}=v_{-n-1}$.

Another example are models of the Kronig-Penney type which have for instance been proposed to describe transport in structured superlattices [30, 31, 32, 33, 34, 35, 36, 37, 38, 39, 40]. Here the operator is defined on continuum wave functions in $L^{2}(\mathbf{R})$ and is given by

$$
H_{K P}=-\frac{d^{2}}{d x^{2}}+V(x)
$$

where $V(x)$ is a step-function describing a sequence of potential barriers of $|\mathcal{A}|$ types that are again arranged consecutively according to the chosen underlying substitution sequence.

As always in the theory of differential operators one is interested in the spectrum of the operators $H$. Let us recall that the spectrum, $\sigma(H)$, of a self-adjoint operator $H$ is defined as the complement of the set of values $E$ for which the resolvent, $(H-E)^{-1}$ is a bounded operator. An important criteria that characterizes the spectrum in the case of Schrödinger operators is that $\sigma(H)$ coincides with the closure of the set of values $E$ for which the time independent Schrödinger equation

$$
H \psi=E \psi
$$


possesses a solution that is polynomially bounded, i.e. for which there exist constants $a$ and $b$ such that $\left|\psi_{E}(n)\right| \leq b|n|^{a}$, for all $n \in \mathbf{Z}$. The values for which $\psi_{E}(n)$ is a square integrable function are called the eigenvalues of $H$, and the closure of the set of eigenvalues is called the point spectrum; the remaining spectrum is the continuous spectrum which can be further decomposed into the absolutely continuous and singular continuous parts, in accordance with the Lebesgue decomposition of the spectral measure. Roughly speaking, the absolutely continuous spectrum is a closed set with non-empty interior, while the singular continuous part ('what remains' of the spectrum after the point and the absolutely continuous parts have been removed) is a Cantor set. The spectral type has important consequences for the transport properties of the models; if the Fermi-energy falls in the absolutely continuous part of the spectrum, we expect a conductor, while if it falls into the point spectrum (or in the complement of the spectrum) one expects to have an insulator. The singular continuous spectrum has generally been considered to be somewhat exotic and is much less understood; however, for the very models we are dealing with here it is quite common and is expected to give rise to interesting new transport phenomena.

Basically since the early papers of Kohmoto et al. it had been conjectured that, at least in the case of the Fibonacci sequence, the operator $H_{t b}$ should have singular continuous spectrum. This was later proven in two remarkable papers by Sütö [12] and (for more general Fibonacci sequences) Bellissard et al. 13. It is a natural question to ask whether this property depends on particular features of the Fibonacci sequence, e.g. on the fact that it is quasi-periodic, and one may pose the question whether and how the spectral type of the Hamiltonian reflects certain features of the sequence, and in particular the nature of the Fourier spectrum of the sequence, or if it could be an indicator for the degree of 'randomness' of the substitution sequence. The most prominent example of a substitution sequence that is not quasiperiodic is certainly the Thue-Morse sequence and this has led to a rather extensive investigation of this example. The question of the spectral type in this case has been discussed in a number of papers 114, 15, 16, 17, 18, 27, 28, 29, 30, 39] both for the tight-binding model and the Kronig-Penney model. In their most recent papers, Ryu et al. 30] arrive at the conclusion that in both cases the spectrum contains an absolutely continuous component. On this basis they argue that the Thue-Morse sequence should be regarded as more 'periodic' than the Fibonacci sequence. Unfortunately, their claim is 
false. In fact, in $\| 18$ (see also [17 for a slightly incomplete argument) it has been proven rigorously that the spectrum of the Thue-Morse model is purely singular continuous (this paper deals mainly with another sequence, the period doubling sequence, but the result applies also to the Thue-Morse case (see the remark following Theorem 3 in [18). In fact, a much more general result on the absence of absolutely continuous spectrum has been obtained in [22] which suggests that the spectral type is quite independent on the particular properties of the substitution sequence. In the next sections we explain those results in more detail and comment in more detail on how the erroneous claims in [30] have been obtained.

\section{Sequences and spectra}

It is a natural question to ask what properties of a given sequence determine the spectral type of the corresponding Schrödinger operator. Classical candidates might appear to be

1. the entropy of a sequence, defined as (see e.g. [4])

$$
I=\lim _{k \uparrow \infty} \frac{1}{k} \ln \#\{\text { different words of length } k \text { occurring in the sequence }\}
$$

2. the Fourier spectrum of the sequence.

However, the entropy of a sequence is clearly too crude a measure; all substitution sequences have zero entropy and are thus not distinguishable from periodic ones. Nonetheless, their spectra are quite different. It is an interesting, and as yet not much investigated question, whether more refined quantities related to the number of different words in a sequence are related to spectral properties. However, again, within the class of substitution sequences this quantity always behaves in the same way and no distinction can be seen.

The Fourier spectrum (see e.g. [41, 42]), on the other hand, varies widely in nature between substitution sequences, ranging all the way from pure point (Fibonacci) over singular continuous (Thue-Morse) to absolutely continuous (Rudin-Shapiro). One might naturally think that this should be reflected in the spectra of the corresponding operators: pure point Fourier should give 
rise to singular continuous spectrum of the Schrödinger operator and absolutely continuous Fourier aught to correspond to point spectrum, with the intermediate singular continuous Fourier somewhere in between. However, there is little evidence to support such conjectures. An attempt to provide a theoretical basis to relate properties of the Fourier spectrum of a sequence to spectral properties of the Schrödinger operators was made by Luck [19] on the basis of perturbation theory. Although his arguments are mathematically heuristic, he succeeded in predicting the scaling of the spectral gaps, at least in cases where the Fourier spectrum is pure point. In other cases, such as Thue-Morse and Rudin-Shapiro, there were no conclusive predictions. This method does demonstrate, however, that a relation between Fourier spectrum and the spectrum of the operator is basically limited to first order perturbation theory, and higher order effects will become relevant if the singularities in the Fourier spectrum are not strong enough.

A more pragmatic point of view would favour the idea that the crucial features of a substitution sequence should reside in its self-similarity which is encoded in its substitution rule. From that point of view, it would seem more natural to conjecture that the spectral type for all substitution sequences (excepting maybe some pathological cases) should be the same, namely singular continuous spectrum. There are a number of sound mathematical results indicating in this direction, with a number of open problems left to close the argument and the remainder of this section is devoted to their explanation.

In all the one dimensional models of the type we consider here, i.e. the tight binding or the Kronig-Penney models, the basic tool of spectral analysis is the transfer matrix formalism. Without entering into the presentation of details that have been presented many times elsewhere (for the KronigPenney model see e.g. [30, 31, 32, 33, 34, 35, 36, 37, 38]) we just note that in all these cases this leads to the investigation of a product of two-by two matrices of the form

$$
P_{n}(E)=\prod_{k=1}^{n} T_{E}\left(u_{n-k}\right)
$$

where $u_{k}$ is the $k$-th letter in the substitution sequence $u$ and $T_{E}: \mathcal{A} \rightarrow$ $S L(2, \mathbf{C})$ is a map that assigns, for fixed energy $E$, to each letter in the alphabet a unimodular two-by-two matrix. The precise form of this map depends, of course, on the specific model and its parameters (such as potential strength, etc.). As we shall see, however, this precise form of $T_{E}$ is irrelevant 
for many qualitative properties of the model such as the spectral type of $H$. Let us also recall that the Lyapunov exponent is defined as

$$
\gamma(E)=\lim _{|n| \rightarrow \infty} \frac{1}{|n|} \ln \left\|P_{n}(E)\right\|
$$

The self-similarity properties of substitution sequences can be used to derive a very efficient method for obtaining crucial information on the asymptotic properties of $P_{n}(E)$. Let for any word $\omega \in \mathcal{A}^{*}$

$$
T_{E}(\omega) \equiv \prod_{\alpha \in \omega} T_{E}(\alpha)
$$

Set moreover, for $k \in \mathbf{N}$,

$$
T_{E}^{(k)}(\omega) \equiv T_{E}^{(k-1)}(\xi(\omega))
$$

with $T_{E}^{(0)} \equiv T_{E}$. Obviously, the quantities $T_{E}^{(k)}(\alpha)$ can then be computed recursively. It has turned out even more useful to derive from these recursion a system of recursive equations for the traces of these transfer matrices, called the trace map. The existence of a trace map has been established first in the case of the Fibonacci sequence by Kohmoto et al. [8], for general substitutions on two-letter alphabets by Allouche and Peyrière 443 and in all generality by Kolár and Nori 44. More recently, there has been considerable effort by several groups to find the simplest form of the trace map [45, 46, 47, but this is not really relevant for the question we are concerned with. We will not enter into the derivation of the trace map (for an exposition see e.g. 22]) but only state that in general there exists a finite subset $\mathcal{B} \subset \mathcal{A}^{*}$ such that if we set $x_{E}^{(k)}(\omega) \equiv \operatorname{tr} T_{E}^{(k)}(\omega)$, then there exist for all $\beta \in \mathcal{B}$ polynomial maps $F_{\beta}: \mathbf{C}^{|\mathcal{B}|} \rightarrow \mathbf{C}$, such that

$$
x_{E}^{(k+1)}(\beta)=F_{\beta}\left(x_{E}^{(k)}\left(\beta_{1}\right), \ldots, x_{E}^{(k)}\left(\beta_{|\mathcal{B}|}\right)\right)
$$

In the specific examples that we consider mostly here, the trace map takes the following form:

1. (Period doubling sequence) $\mathcal{B}=\mathcal{A}$, and

$$
\begin{aligned}
& x_{E}^{(k+1)}(a)=x_{E}^{(k)}(a) x_{E}^{(k)}(b)-2 \\
& x_{E}^{(k+1)}(b)=x_{E}^{(k)}(a) x_{E}^{(k)}(a)-2
\end{aligned}
$$


2. (Thue-Morse sequence) Here it is useful to use slightly more complicated variables. We set $x_{E}^{(k)}=\operatorname{tr} T_{E}^{(k)}(a)$ and $v_{E}^{(k)}=\frac{1}{2} \operatorname{tr}\left(T_{E}^{(k)}(a)-T_{E}^{(k)}(b)\right)^{2}$ and finally $w_{E}^{(k)}=v_{E}^{(k)}+4-\left(x_{E}^{(k)}\right)^{2}$. Then 16

$$
x_{E}^{(k+1)}=2-w_{E}^{(k)}, \quad w_{E}^{(k+1)}=\left(x_{E}^{(k)}\right)^{2} w_{E}^{(k)}
$$

These trace maps are universally used tools to investigate these models. They do depend only on the substitution sequence and not on the specific choice of the model or its parameters which enter only through the initial conditions in a straightforward manner.

To use the trace map in the spectral analysis we need to relate the spectrum to quantities related to the dynamical system given by the trace map. This quantity will be, naturally, the stable set of the dynamical system: we should expect that a particular value of the energy, $E$, is in the spectrum of the Hamiltonian, if and only if the corresponding vector of initial traces $x_{E}^{(0)}$ remains bounded under subsequent applications of the trace map.

However, this point turns out to be quite subtle and represents, in fact, the main difficult part in the determination also of the spectral type. First of all, it is quite easy to give a precise definition of a stable set which guarantees that it will contain the spectrum: we say that its complement, called the unstable set, is the set of initial traces $x_{E}^{(0)}$ with the property that there exists an $n_{0}$ large enough such that for all $n>n_{0}$, the images under $n$ applications of the trace map have first component, $x_{E}^{(n)}\left(\alpha_{0}\right)$ whose absolute value is larger than two (for a precise formal definition see e.g. [22]). The point is that under this condition, we can construct a sequence of periodic approximants of $H$, converging strongly to $H$, for which we know by Floquet theory that $E$ is in the interior of a spectral gap, and this implies that the same holds for the limiting operator. Note that this definition does not imply that in the stable set the traces remain bounded; it suffices that there exists an infinite number of values $n$ for which the first trace gets smaller than two! The period doubling sequence furnishes an example in which such behaviour actually occurs, i.e. for some certain values of the energy the traces undergo fluctuations with ever growing amplitudes, although, and this is a general characteristic feature, the growth is slower than exponential (see [22]). This can easily be deduced from the results presented in [18]. 
So what about the converse statement? Intuitively, one is tempted to believe that if the trace of the transfer matrix has modulus less than two, this should imply that all solutions of both the homogeneous Schrödinger equation (7) and the inhomogeneous equation for the Green's function

$$
(H-E) G=\delta_{0}
$$

where $\delta_{0}$ denotes the delta-function concentrated at zero, cannot tend to zero at infinity and in particular are not square-summable (recall that the transfer matrices have determinant one; thus if their trace is less than or equal to two, both their eigenvalues have modulus one!) which implies first that $E$ is not an eigenvalue and second that $E$ is in the spectrum of $H$. However, a moment's reflection will show that this argument is premature and more work is needed to justify it (the point being that no information is given on the eigenvectors of the transfer matrices, the angle between which could tend to zero in which case a decaying solution cannot be excluded). Still, this argument has been made rigorous in some examples, namely the Fibonacci sequences, the Thue-Morse sequence and the period-doubling sequence. However, already in the period-doubling case, this required a fairly cumbersome analysis that appeared to be impossible to carry through in more complicated situations.

A less direct, but technically more feasible approach is based on the following observation: from quite general argument, it is known that the spectrum of a Schrödinger operator must always contain the set of energies for which the Lyapunov exponent is zero (the converse being false in general; for random sequences, the Lyapunov is strictly positive even in the spectrum). Thus, if one can show that the Lyapunov exponent is zero for all energies in the stable set, one can close the circle of inclusions and shows that all three sets are identical! This idea was shown to work in [22] for a very large class of substitution sequences, namely those giving rise to so-called "semi-primitive" trace maps (for a definition see [22]).

Apart from its generality, an advantage of this method is that it yields as a by-product immediately the singular nature of the spectrum. By a result of Kotani [48] (augmented by some simple soft analysis that can be found in [18]) it is known that, for any substitution sequence (excepting the trivial case of periodic sequences), the set of energies on which the Lyapunov exponent vanishes must be of zero Lebesgue measure. Thus, the presence of absolutely continuous spectrum is immediately excluded in all these cases! We would 
like to stress that all these argument apply in the Kronig-Penney models just as in the tight binding models, even though the theorems in the original litterature were stated only for the latter case. The problem left open by this method is that of the possible existence of eigenvalues. They are excluded under assumptions which allow to show that the first method works but are certainly too restrictive. It is quite likely that in fact the spectrum will be singular continuous (i.e. of measure zero and no eigenvalues) whenever it coincides with the stable set of the trace map, but some new idea is needed to prove this. The other main open problem is of course to know whether the assumption of a semi-primitive trace map is really necessary for the absence of absolutely continuous spectrum. There is one example, the Rudin-Shapiro sequence, for which the trace map is not semi-primitive, but unfortunately, there are also no very definite results on the nature of the spectrum in this case. Dulea et al. [49 have presented some evidence based on a scaling analysis and numerical data that for strong potentials, the spectrum should be pure point. This would be very interesting, if it can be confirmed. But of course, all such results should be regarded with great caution.

\section{$3 \quad$ Extended states and spectra}

We have seen in the last section that the question of the nature of the spectra in our models is settled rigorously for a large class of substitution sequences and in particular, with great detail, in the case of the Thue-Morse sequence. It is still interesting to analyze why and how the authors of [30] arrived at the false conclusion that in this example there exists an absolutely continuous component in the spectrum. The main basis of their analysis resides in the distinction of (generalized) eigenstates as 'extended' or 'critical'. Now, in the Thue-Morse case (and in fact in all examples where singular continuity of the spectrum was proven) it turns out that for all values of the energy in the spectrum, the solutions of the initial value problem $(H-E) \psi=0$ do not tend to zero at infinity. Thus, reasonably, all states might be called 'extended'. Still, there are quite different ways a function that does not tend to zero may behave. A particularly nice one would be to be periodic, or, at least, to be a possibly aperiodic repetition of several patterns of constant

length. This latter case occurs quite typically for substitution potentials and 
it is apparently this behaviour that is referred to as 'extended states'. While this has nothing whatsoever to do with the presence or absence of absolutely continuous spectrum, this is an interesting phenomenon and deserves some comments.

Let us for simplicity consider the case of a two-letter alphabet, say $\mathcal{A}=$ $\{a, b\}$. Now it may happen that for given integer $k$ and for special values of the energy $E$ the matrices $T_{E}^{(k)}(a)$ and $T_{E}^{(k)}(b)$ commute. Then the two matrices will posses at least one common eigenvector, and this will give rise to a solution of the Schrödinger equation whose behaviour over steps of length $\left|\xi^{k}(a)\right|$, resp. $\left|\xi^{k}(b)\right|$, we can easily trace. If one of the traces of the two matrices is larger than two, there are in fact two such solutions which will grow either to the left or to the right exponentially fast, so we are out of the spectrum. The interesting case arises when the two traces have modulus less than or equal to two: in this case they just multiply the eigenvector by a phase and we obtain an extended solution whose behaviour is particularly simple. Up to the phase factor, it consists of two patterns of length $\left|\xi^{k}(a)\right|$ and $\left|\xi^{k}(b)\right|$ which alternate according to the way the letters $a$ and $b$ are arranged in the substitution sequence itself.

In general it may be expected that the number of values $E$ for which this phenomenon occurs should increase with $k$ : the entries of the corresponding matrices are polynomials of ever higher degree in $E$. Of course, the details here depend on the particular model via the dependence of the basic transfer matrices on the energy. In the Thue-Morse case and in the period-doubling case, it has previously been noticed that for each $k$ there exist $2^{k-1}$ values of $E$ for which such solutions exist (see [18, 17] and [18] resp.). Their occurrence in both sequences shows in particular that they are not tied to the question of quasiperiodicity of the sequence, as appears to be believed in [30]. It should be noted that the case of the Fibonacci sequence is quite peculiar, as there the matrices $T_{E}^{(k)}(a)$ and $T_{E}^{(k)}(b)$ can only commute if $T_{E}^{(0)}(a)$ and $T_{E}^{(0)}(b)$ commute (this is readily proven by induction; the crucial point is that $A$ commutes with $A B$ if and only if $A$ commutes with $B$ ) which, at least in the tight binding model, is only true in the trivial case where $v \equiv 0$.

In any case, it should be kept in mind that the energies at which such solutions exist always form at most a countable set. The continuous spectrum, however, is by its definition uncountable. Therefore, they can never be significant for absolutely continuous spectrum, and statements like 'one 
half of the states are extended' are quite meaningless.

We should stress, however, that the properties of generalized eigenfunctions will be quite important for other physical, in particular transport properties of these systems (see e.g. [50, 51]) even though this entire field is not yet sufficiently investigated. We would like to mention in this context that the types of extended states we have discussed above occurs also in certain cases of random potentials with constraints. An example that was discussed extensively in recent years is the 'random dimer model' (see [52, 53]) introduced to describe certain anomalous conductivity properties of some polymers.

\section{Conclusions}

We have explained in the preceeding sections that for a large class of models based on substitution sequences the spectrum has no absolutely continuous component and moreover is concentrated on a set of zero Lebesgue measure. Moreover, the spectrum coincides in all these cases with the set of energies for which the Lyapunov exponent is equal to zero (contrary to what is stated for instance in [39]). For some of the most studied examples, namely the Fibonacci sequences, the Thue-Morse sequence and the period-doubling sequence, it has moreover been shown that no eigenvalues can exist and therefore the spectrum is purely singular continuous in these cases. There appears to be no connection between this fact and the nature of the Fourier spectrum of the sequences.

There are a number of important problems that remain, however, open. First, we would like to see whether the absence of absolutely continuous spectrum can be proven for all aperiodic substitution sequences. We expect this to be true. Second, it would be interesting to see whether there are examples of substitution sequences which give rise to point spectrum. A candidate is here the Rudin-Shapiro sequence and it is well worth-while to study it more thoroughly.

Further questions regard the more detailed structure of the spectra, beyond just the spectral type. Only in the Thue-Morse and the period-doubling cases is a very detailed description of the spectrum available (e.g. precise asymptotics for the behaviour of all spectral gaps). It is a quite bothering feature that even in the simplest case of all, the golden Fibonacci sequence, 
the opening of the gaps at small potentials is not known! In this context it would be very interesting to give a more rigorous foundation to the perturbation theoretic arguments of Luck [19]. Also one would like to see more precise relations between characteristics of the spectra (e.g. fractal and correlation dimensions of the spectral measure) and actual transport properties. At least from a mathematical point of view, there is little more than some vague ideas that exist so far, and we do not want to discuss this point here.

Finally, all results so far are obtained for models that are associated to products of two-by-two matrices (excepting the gap-labelling theorems [20, 21] which are valid for much larger classes of systems). Thus models with long-range hopping models on a strip are not covered by existing theorems. The reason for this is basically that there is no analogue of the trace map known in these cases. This leaves a lot of room for further investigations.

\section{References}

[1] Shechtman, D., Blech, I., Gratias, D., Cahn, J.V.: Metallic phase with long-range orientational order and no translational symmetry, Phys. Rev. Lett. 53, 1951-1953 (1984)

[2] see e.g. Steinhardt, P.J., Ostlund, S.: The physics of quasicrystals, Singapore, Philadelphia: World Scientific 1987;

Hof, A.: Quasi-crystals, aperiodicity and lattice systems, Thesis Groningen 1992;

Dulea, M.: Physical properties of one-dimensional deterministic aperiodic systems, Thesis

Linköping 1992

[3] Dekking, F.M.: Combinatorial and statistical properties of sequences generated by substitutions, Thesis Amsterdam 1980

[4] Queffélec, M.: Substitution dynamical systems. Spectral Analysis, Lecture Notes in Mathematics 1294, Berlin, Heidelberg, New-York: Springer 1987

[5] Luck, J.-M.: Critical behavior of the aperiodic quantum Ising chain in a transverse magnetic field, J. Stat. Phys. 72, 417-458 (1993) 
[6] Grimm, U., Baake, M.: Non-periodic Ising quantum chains and conformal invariance, Preprint Melbourne University 24-1993, to appear in J. Stat. Phys.;

Baake, M., Grimm, U, Pisani, C.: Partition function zeros for aperiodic systems, Preprint Universität Stuttgart itap-11/93-2

[7] Combescure, M.: Recurrent versus diffusive dynamics for a kicked quantum sytem, J. Stat. Phys. 62, 779-791 (1991);

Combescure, M.: Recurrent versus diffusive dynamics for a kicked quantum oscillator, Ann. Inst. Henri Poincaré 57, 67-87 (1992)

[8] Kohmoto, M., Kadanoff, L.P., Tang, C.: Localization problem in one dimension: Mapping and escape, Phys. Rev. Lett. 50, 1870-1872 (1983)

[9] Ostlund, S., Pandit, R., Rand, D., Schellnhuber, H.J., Siggia, E.D.: Schrödinger equation with an almost periodic potential, Phys. Rev. Lett. 50, 1873-1876 (1983);

[10] Kohmoto, M., Oono, Y.: Cantor spectrum for an almost periodic Schrödinger equation and a dynamical map, Phys. Lett. 102A, 145-148 (1984)

[11] Casdagli, M.: Symbolic dynamics for the renormalization map of a quasiperiodic Schrödinger equation, Commun. Math. Phys. 107, 295318 (1986)

[12] Sütő, A.: The spectrum of a quasiperiodic Schrödinger operator, Commun. Math. Phys. 111, 409-415 (1987);

Sütö, A.: Singular continuous spectrum on a Cantor set of zero Lebesgue measure for the Fibonacci hamiltonian, J. Stat. Phys. 56, 525-531 (1989)

[13] Bellissard, J., Iochum, B., Scoppola, E., Testard, D.: Spectral properties of one dimensional quasi-crystals, Commun. Math. Phys. 125 , 527-543 (1989)

[14] Axel, F., Allouche, J.-P., Kléman, M., Mendès France, M., Peyrière, J.: Vibrational modes in a one-dimensional "quasi alloy", the Morse case, J. de Phys. Colloq. 47 C3, 181-187 (1986) 
[15] Cheng, Z., Savit, R., Merlin, R.: Structure and electronic properties of Thue-Morse lattices, Phys. Rev. B37, 4375-4382 (1988)

[16] Bellissard, J.: Spectral properties of Schrödinger's operator with a ThueMorse potential, in "Number theory and physics", Luck, J.-M., Moussa, P., Waldschmidt, M. (eds.), Springer proceedings in physics 47, Berlin, Heidelberg, New York: Springer 1990, pp. 140-150

[17] Axel, F., Peyrière, J.: Spectrum and extended states in a harmonic chain with controlled disorder: effects of the Thue-Morse symmetry, J. Stat. Phys. 57, 1013-1047 (1989)

[18] Bellissard, J., Bovier, A., Ghez, J.-M.: Spectral properties of a tight binding hamiltonian with period doubling potential. Commun. Math. Phys. 135, 379-399 (1991)

[19] Luck, J.-M.: Cantor spectra and scaling of gap widths in deterministic aperiodic systems, Phys. Rev. B39, 5834-5849 (1989)

[20] Bellissard, J.: Gap labelling theorems for Schrödinger's operators. in "From number theory to physics", Waldschmidt, M., Moussa, P., Luck, J.-M., Itzykson, C. (eds.), Berlin, Heidelberg, New York: Springer 1992, pp. $538-630$

[21] Bellissard, J., Bovier, A., Ghez, J.-M.: Gap labelling theorems for one dimensional discrete Schrödinger operators, Rev. Math. Phys. 4, 1-37 (1992)

[22] Bovier, A., Ghez, J.-M.: Spectral properties of one-dimensional Schrödinger operators with potentials generated by substitutions, Commun. Math. Phys. 158, 45-66 (1993)

[23] Merlin, R., Bajema, K., Clarke, R., Juang, F.-Y., Bhattacharya, P.K.: Quasi-periodic GaAs-AlAs heterostructures, Phys. Rev. Lett. 55, 17681770 (1985);

Todd, J., Merlin, R., Clarke, R., Mohanty, K.M., Axe, J.D.: Synchrotron X-ray study of a Fibonacci superlattice, Phys. Rev. Lett. 57, 1157-1160 (1986);

Bajema, K., Merlin, R.: Raman scattering by acoustic phonons in Fibonacci GaAs-AlAs superlattices, Phys. Rev. B36 4555-4557 (1987); 
Merlin, R., Bajema, K.: Raman scattering by acoustic phonons and structural properties of Fibonacci, Thue-Morse and random superlattices, J. Phys. Colloq. (France) 48 C5, 503-506 (1987)

[24] Axel, F., Terauchi, H.: High-resolution X-ray diffraction spectra of Thue-Morse GaAs-AlAs heterostructures: Towards a novel description of disorder, Phys. Rev. Lett. 66, 2223-2226 (1991)

[25] Bellissard, J., Bovier, A., Ghez, J.-M.: Discrete Schrödinger operators with potentials generated by substitutions, in "Differential Equations with Applications to Mathematical Physics", Ames, W.F., Harrell, E.M., Herod, J.V. (eds.), Boston: Academic Press 1993, pp. 13-23

[26] Bovier, A., Ghez, J.-M. Schrödinger operators with substitution potentials. in "Cellular Automata and Cooperative Systems", Boccara, N., Goles, E., Martinez, S., Picco, P. (eds.) Dordrecht: Kluwer Academic Press 1993, pp. 67-83

[27] Riklund, R., Severin, M., Youyan Liu: The Thue-Morse aperiodic crystal, a link between the Fibonacci quasicrystal and the periodic crystal, Int. J. Mod. Phys. B1, 121-132 (1987)

[28] Ma, H.R., Tsai, C.H.: On the energy spectra of one-dimensional quasiperiodic systems, J. Phys. C: Solid State Phys. 21, 4311-4324 (1988);

Qin, M.G., Ma, H.R., Tsai, C.H.: A renormalisation analysis of the onedimensional Thue-Morse aperiodic chain, J. Phys.: Conden. Matter 2, 1059-1072 (1990)

[29] La Rocca, G.C.: Phase transition in the multifractal spectrum of the Thue-Morse lattice, Solid State Commun. 70, 115-118 (1989)

[30] Ryu, C.S., Oh, G.Y., Lee, M.H.: Extended and critical wave functions in a Thue-Morse chain, Phys. Rev. B46, 5162-5168 (1992);

Ryu, C.S., Oh, G.Y., Lee, M.H.: Electronic properties of a tight- binding and a Kronig-Penney model of the Thue-Morse chain, Phys. Rev. B48, 132-141 (1993);

Oh, G.Y., Lee, M.H.: Band-structural and Fourier-spectral properties of one-dimensional generalized Fibonacci lattices, Phys. Rev. B48, 1246512477 (1993) 
[31] Kollár, J., Sütő, A.: The Kronig-Penney model on a Fibonacci lattice, Phys. Lett. A117, 203-209 (1986)

[32] Kalugin, P.A., Kitaev, A.Y., Levitov, L.S.: Electron spectrum of a onedimensional quasicrystal, Sov. Phys. JETP 64, 410-416 (1986)

[33] Hu, P., Ting, C.S.: Electron transmission in a one-dimensional quasicrystal, Phys. Rev. B34, 8331-8334 (1986)

[34] MacDonald, A.H., Aers, G.C.: Continuum-model acoustic and electronic properties for a Fibonacci superlattice, Phys. Rev. B36, 9142-9145 (1987)

[35] Würtz, D., Soerensen, M.P., Schneider, T.: Quasiperiodic KronigPenney model on a Fibonacci superlattice, Helv. Phys. Acta 61, 345-362 (1988)

[36] Holzer, M: Three classes of one-dimensional, two-tile Penrose tilings and the Fibonacci Kronig-Penney model as a generic case, Phys. Rev. B38, 1709-1720 (1988)

[37] Kim, Y.-J., Oh, G.Y., Lee, M.H.: Electronic structure of a onedimensional quasicrystal, J. Korean Phys. Soc. 22 136-140 (1989)

[38] Thomas, U., Kramer, P.: The Fibonacci quasicrystal reconsidered: variety of energy spectra for continuous Schrödinger equations with simple potentials, Int. J. Mod. Phys. B3, 1205-1235 (1989);

Baake, M., Joseph, D., Kramer: Periodic clustering in the spectrum of quasiperiodic Kronig-Penney models, Phys. Lett. A168, 199-208 (1992)

[39] Avishai, Y., Berend, D.: Transmission through a one-dimensional sequence of $\delta$-function potentials, Phys. Rev. B41, 5492-5499 (1990);

Avishai, Y., Berend, D.: Transmission through a Thue-Morse chain, Phys. Rev. B45, 2717-2724 (1992)

[40] Ghosh, P.K.: The Kronig-Penney model on a generalized Fibonacci lattice, Phys. Lett. A161, 153-157 (1991)

[41] Godrèche, C., Luck, J.-M.: Multifractal analysis in reciprocal space and the nature of the Fourier transform of self-similar structures, J. Phys. 
A: Math. Gen. 23, 3769-3797 (1990);

Luck, J.-M., Godrèche, C., Janner, A, Janssen, T.: The nature of the atomic surfaces of quasiperiodic self-similar structures, J. Phys. A: Math. Gen. 26, 1951-1999 (1993) and references therein

[42] Kolar, M., Iochum, B., Raymond, L.: Structure factor of $1 D$ systems (superlattices) based on two-letter substitution rules. I. $\delta$ (Bragg) peaks, J. Phys. A: Math. Gen. 26, 7343-7366 (1993) and references therein

[43] Allouche, J.-P., Peyrière, J.: Sur une formule de récurrence sur les traces de produits de matrices associés à certaines substitutions $\mathrm{C}$. R. Acad. Sci. Paris 302 serie II, 1135-1136 (1986)

[44] Kolár, M., Nori, F.: Trace maps of general substitutional sequences, Phys. Rev. B42, 1062-1065 (1990)

[45] Peyrière, J.: On the trace map for products of matrices associated with substitutive sequences, J. Stat. Phys. 62, 411-414 (1991);

Peyrière, J., Wen Zhi-ying, Wen Zhi-xiong: Polynômes associés aux endomorphismes de groupes libres, L'Enseignement Math. 39 (1993)

[46] Avishai, Y., Behrend, D., Glaubman, D.: Minimum-dimension trace maps for substitution sequences, Preprint Beer-Sheva University (1993)

[47] Baake, M., Grimm, U., Joseph, D.: Trace maps, invariants, and some of their applications, Int. J. Mod. Phys. B7, 1527-1550 (1993)

[48] Kotani, S.: Jacobi matrices with random potentials taking finitely many values, Rev. Math. Phys. 1, 129-133 (1990)

[49] Dulea, M., Johansson, M., Riklund, R.: Unusual scaling of the spectrum in a deterministic aperiodic tight-binding model, Phys. Rev. B47, 85478551 (1993)

[50] Sutherland, B., Kohmoto, M.: Resistance of a one-dimensional quasicrystal, Phys. Rev. B36, 5877-5886 (1987)

[51] Iochum, B., Testard, D.: Power law growth for the resistance in the Fibonacci model, J. Stat. Phys. 65, 715-723 (1991);

Iochum, B., Raymond, L., Testard, D.: Resistance of one-dimensional quasicrystals, Physica A187, 353-368 (1992) 
[52] Dunlap, D.H., Kundo, K., Phillips, P.W.: Absence of localization in certain statistically disordered lattices in any spatial dimension, Phys. Rev. B40, 10999-11006 (1989);

Dunlap, D.H., Wu, H.-L., Phillips, P.W.: Absence of localization in a random-dimer model, Phys. Rev. Lett. 65, 88-91 (1990)

[53] Bovier, A.: Perturbation theory for the random dimer model, J. Phys. A: Math. Gen. 25, 1021-1029 (1992) 